\title{
Exploring cancer development in adulthood: cholinesterase depression and genotoxic effect from chronic exposure to organophosphate pesticides among rural farm children
}

\begin{abstract}
Children are the vulnerable group in the agricultural community due to their early exposure to pesticides through the dynamic interplay between genetic predisposition, environment, and host-related factors. This study aims to identify the possible association between the depression in blood cholinesterase level and genotoxic effect among farm children. The results of micronuclei assay and comet assay showed that the reduced blood cholinesterase level from organophosphate pesticide exposure is significantly associated with an increase in chromosome breakage and DNA strand breaks. These genotoxicity end points suggest that farm children's cells experience early DNA damage that may lead to uncontrolled cell proliferation during their adulthood. Thus, farm children who grow up near pesticide-treated farmland have a higher probability of developing cancer than children with minimal or zero exposure to pesticides.
\end{abstract}

Keyword: Children; Cholinesterase depression; Genotoxicity risk; Organophosphate 